\title{
Numerical Study of Low-Speed Droplets Impacting on the Fabric Surface
}

\author{
Qilei GUO*, Peng SUN**, Chen CHEN***, Yao MA**** \\ *Civil Aviation Flight University of China, Guanghan 618307, China, E-mail: cafuc_guoql@outlook.com \\ **Civil Aviation Flight University of China, Guanghan 618307, China, E-mail: stewen1981@163.com \\ ***Civil Aviation Flight University of China, Guanghan 618307, China, E-mail: chenchen_echo@163.com \\ ****Civil Aviation Flight University of China, Guanghan 618307, China, E-mail: cafucmy@ 126.com \\ crossref http://dx.doi.org/10.5755/j01.mech.25.6.22947
}

\section{Introduction}

The droplet impact can be very significant in various engineering applications, ranging from inkjet printing, engine washing, to aircraft icing [1-4]. Specially, droplet impact has also a great effect on parachute working process. In severe weather, the supercool droplet could even cause local icing to degrade the decelerating of parachute and accordingly reduce parachute success. Hence, it is of great academic and engineering significance to study the droplet impacting on fabric surfaces.

Scholars have made fruitful achievements about the droplet impacting on impermeable surfaces. Shen and Bi [5] studied the effects of several dimensionless parameters on the deformation of a droplet impacting on a $2 \mathrm{D}$ round surface by using lattice Boltzmann algorithm and revealed that initial impacting velocity plays a significant role in impact dynamic. Li and Zhang [6-7] experimentally and numerically measured the impact forces of the low-speed water droplet colliding on a light and tiny aluminum plate and investigated the effects of impact velocity and diameter of droplets, Reynolds and Weber numbers on the impact forces. Blake and Thompson [8] employed an enthalpy-porosity method to numerically simulate the impact and solidification of a super-cooled water droplet on a cooled substrate.

However, the study of impacting droplets spreading over permeable media has received little attention. Reis et al [9] applied magnetic resonance imaging (MRI) techniques to obtain the evolving shape of the liquid droplet inside the porous medium during evaporation. Then Reis [10] employed Finite Volume Method (FVM) to simulate the fluid dynamics of impacting droplet onto a porous medium. Golpaygan et al [11] proved that the droplet spreading inside a porous substrate is governed by the We number of the impacted droplet, while the major penetration is decided by the Re number. Kim and Lee [12] applied the volume of fluid (VOF) method to simulate the effects of porosity on the drop motion and examined the effect of the three-phase contact angle line by varying adjusting parameters. Choi and Son [13-14] employed the level-set (LS) method to track the droplet deformation and investigated the effects of initial droplet radius, impact velocity, contact angle and porosity on the droplet spreading and penetration in a porous medium. Cheng [15] investigated the droplet impacting on canopy fabric surface by experiments and revealed that the impact velocity is the most crucial factor in droplet spreading.

The droplet impinging on the porous medium is controlled by two processes, which are the droplet spreading on the surface and an infiltration inside the porous medium [16]. The fabric is a porous medium, and its characteristics make the impact process between permeable and impermeable surfaces. However, the existing numerical research results have not made an accurate and rigorous analysis of the phenomena that droplets impact on the fabric surface. The experimental research hardly obtained the pressure field and velocity distribution, due to the limitations of experimental method.

In this work, the numerical model of the fluid dynamics of liquid droplets impacting on the fabric surface with VOF method was proposed. The numerical results obtained by the proposed model were compared to experimental results. The mechanism of the liquid droplet morphological evolution was investigated by pressure distribution and velocity vector, and the obvious bubble entrapment was captured. The evolution laws of the spreading factor and the contact angle at the liquid-porous interface were investigated and the reason why the contact angle is dynamic is analyzed. The effects of droplet diameter and impact velocity on the fluid flow characteristics are also discussed.

\section{Mathematical modelling}

It is important to define the computational domain and the initial conditions, before introducing the governing equations. In this work, the two-dimensional axisymmetric model is used, as shown in Fig. 1.

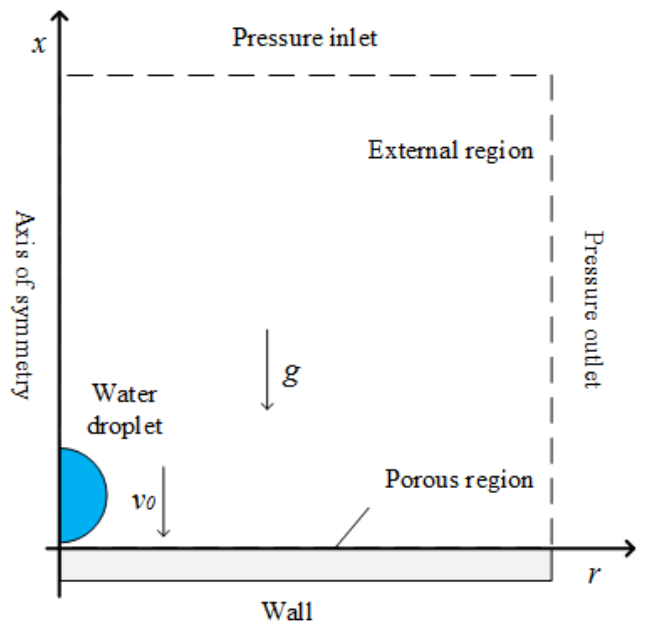

Fig. 1 The configuration of the computational domain

The computational domain is defined to the $16 \mathrm{~mm}$ for the length and height, respectively. It is remarkable that a thin slice region at the bottom of the whole domain is the 
porous region. The liquid droplet is set in a tiny distance above the porous region, with an initial impact velocity. Under the force of gravity, the liquid droplet flows downward and impacts on the porous surface.

In the approach, the following assumption are made as: 1. the flow is axisymmetric, laminar and Newtonian fluid; 2. the properties of liquid, air and solid are constant during the numerical simulation.

\subsection{Governing equations in external region}

The conservation equations of mass and momentum in external region can be expressed as:

$$
\begin{aligned}
& \nabla \mathbf{U}=0, \\
& \frac{\partial(\rho \mathbf{U})}{\partial t}+\nabla(\rho \mathbf{U U})=\nabla \mathbf{T}+\rho \mathbf{g}+\sigma \kappa \nabla \gamma,
\end{aligned}
$$

where: $\mathbf{U}$ is the velocity vector; $\rho$ is the constant density of the fluid; $\mathbf{g}$ is the acceleration due to gravity; $\sigma$ is the surface tension; $t$ is time, $\mathbf{T}$ can be written for a Newtonian fluid as $\mathbf{T}=-p \mathbf{I}+\mu \nabla \mathbf{U}+\mu(\nabla \mathbf{U})^{T}$, which is called to the stress tensor, $p$ is the pressure, $\mu$ is the kinetic viscosity of the fluid, $\kappa$ is the interface curvature and $\gamma$ is the indicator function, which are respectively determined by following expression:

$$
\kappa=-\nabla\left(\frac{\nabla \gamma}{|\nabla \gamma|}\right)
$$

$$
\gamma=\left\{\begin{array}{l}
0, \quad \text { for points belonging to one phase; } \\
0<\gamma<1, \text { for points at the phase-interface; } \\
1, \quad \text { for points belonging to another phase }
\end{array}\right.
$$

\subsection{Governing equations in porous region}

It is noteworthy that the conservation equations of mass and momentum in porous region with a porosity $\varepsilon$, which is treated as a constant in this approach, can be expressed as $[9,10,17]$ :

$$
\begin{aligned}
& \nabla\langle\mathbf{U}\rangle=0, \\
& \frac{\partial(\rho\langle\mathbf{U}\rangle)}{\partial t}+\frac{1}{\varepsilon} \nabla(\rho\langle\mathbf{U}\rangle\langle\mathbf{U}\rangle)= \\
& =\nabla \mathbf{T}+\rho \mathbf{g} \cdot \varepsilon+\varepsilon \sigma \kappa \nabla \gamma-B \varepsilon\langle\mathbf{U}\rangle,
\end{aligned}
$$

where: $\langle\mathbf{U}\rangle$ is the Darcy velocity vector, also called the macroscopic averaged velocity vector in porous medium, $\langle\mathbf{U}\rangle=\varepsilon \mathbf{U}, \varepsilon$ is the porosity of the fabric medium, which can be written as $\varepsilon=\frac{V_{P}}{V}, V_{P}$ is the volume of pores in porous medium, $V$ is the total volume of the porous material [18]. The drag coefficient B is defined as [19]:

$$
B=\frac{150 \mu(1-\varepsilon)^{2}}{\varepsilon^{2} d_{p}{ }^{2}}+\rho \frac{1.75 \mu \varepsilon|\langle\mathbf{U}\rangle|}{\varepsilon^{2} d_{p}},
$$

here: $d_{p}$ represents the particle diameter in the porous medium.

2.3. Combined governing equations for the whole domain

As above, the governing equations in this approach are determined, and it is necessary to link the equations for the fluid flow outside and inside the porous region. The two sets of governing equations in the external and porous region are similar, differing only in the additional resistance term and the appearance of the porosity, which must comply with the matching conditions at the porous-fluid interface. The boundary conditions at the porous-fluid interface can be summarized as [14, 20]:

$$
\mathbf{U}=\langle\mathbf{U}\rangle, \quad p=\langle p\rangle, \frac{\partial \mathbf{U}}{\partial x}=\frac{\partial\langle\mathbf{U}\rangle}{\partial x} .
$$

Therefore, by introducing the following effective velocity vector $\hat{\mathbf{U}}$ and pressure $\hat{p}$, which are continuous through the porous-fluid interface,

$$
\hat{\mathbf{U}}=\gamma \mathbf{U}+(1-\gamma)\langle\mathbf{U}\rangle, \hat{p}=\gamma p+(1-\gamma)\langle p\rangle
$$

the combined government equations complying with the matching conditions can be written as:

$$
\nabla \hat{\mathbf{U}}=0
$$

$\frac{\partial(\hat{\rho} \hat{\mathbf{U}})}{\partial t}+\frac{1}{\hat{\varepsilon}} \nabla(\hat{\rho} \hat{\mathbf{U}} \hat{\mathbf{U}})=\nabla \hat{\mathbf{T}}+\hat{\rho} \mathbf{g} \hat{\varepsilon}+\hat{\varepsilon} \sigma \kappa \nabla \gamma-\hat{B} \hat{\mathbf{U}}$,

where: $\hat{\varepsilon}, \hat{\mathbf{T}}$ and $\hat{B}$ are defined as $\hat{\mathbf{U}}$ and $\hat{p}$ by following expressions:

$$
\begin{aligned}
& \hat{\varepsilon}=\gamma+(1-\gamma) \varepsilon \\
& \hat{\mathbf{T}}=\gamma \mathbf{T}+(1-\gamma)\langle\mathbf{T}\rangle \\
& \hat{B}=\frac{150 \hat{\mu}(1-\varepsilon)^{2}}{\varepsilon^{2} d_{p}{ }^{2}}+\rho \frac{1.75 \hat{\mu} \varepsilon|\hat{\mathbf{U}}|}{\varepsilon^{2} d_{p}},
\end{aligned}
$$

\section{Numerical results and discussion}

\subsection{Morphological evolution of the impacting droplet}

The liquid properties used in the approach and the whole 16 initial impact conditions are presented in Table 1. In order to study the dynamic characteristics of the droplet impacting on the fabric surface and compare to experiments results, we regard the model of the impacting droplet with the $2.12 \mathrm{~mm}$ diameter and $0.75 \mathrm{~m} / \mathrm{s}$ velocity as the benchmark model.

As demonstrated in Fig. 2, numerical results of the benchmark model are in well agreement with experimental results [15]. Both of them have three stages: Spreading Stage, Recoiling Stage and Oscillating Stage. In spreading 
stage (0-2.7 ms), the upper part of the droplet is spherical and the lower part spreads to the periphery. The wetting area between droplet and fabric surface spreads to the maximum diameter and the droplet shape is similar to a bowl. Under the action of surface tension, the droplet begins to recoil in the opposite direction and enter in recoiling stage (2.7$9 \mathrm{~ms}$ ). In this stage, the wetting area almost keep the original state without increasing. However, it can be observed that the droplet concentrates to the center and a little bead separates from the body of droplet due to inertia. In oscillating stage (9-70 ms), the droplet rapidly changes from conical to hemispherical due to surface tension, and keeps oscillating. After the bead falls down into the liquid body, the droplet eventually tends to stabilize.

Table1

Properties of water droplet at $20^{\circ} \mathrm{C}$

\begin{tabular}{|c|c|c|c|c|c|c|c|}
\hline Liquid & \multicolumn{3}{|c|}{ Properties at $20^{\circ} \mathrm{C}$} & \multicolumn{3}{c|}{ Impact conditions } \\
\hline \multirow{3}{*}{ Water } & $\begin{array}{c}\text { Density, } \\
\mathrm{kg} / \mathrm{m} 3\end{array}$ & $\begin{array}{c}\text { Viscosity, } \\
\mathrm{mPa} \mathrm{s}\end{array}$ & $\begin{array}{c}\text { Surface } \\
\text { tension, } \\
\mathrm{mN} / \mathrm{m}\end{array}$ & Velocity, $\mathrm{m} / \mathrm{s}$ & Diameter, $\mathrm{mm}$ & Re & We \\
\cline { 2 - 8 } & 998.2 & 1.0 & 72.8 & $0.75 / 1.39 / 1.94 / 2.42$ & $2.12 / 2.98 / 3.42 / 4.54$ & $1587-10965$ & $16.35-364.50$ \\
\hline
\end{tabular}

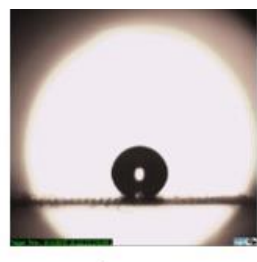

$0 \mathrm{~ms}$

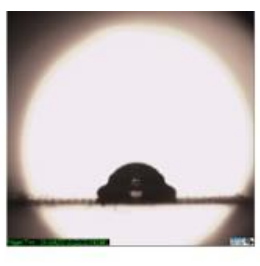

$1 \mathrm{~ms}$

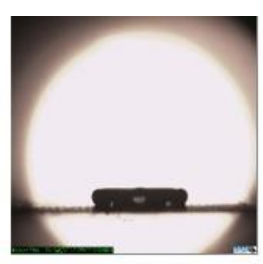

$2.67 \mathrm{~ms}$

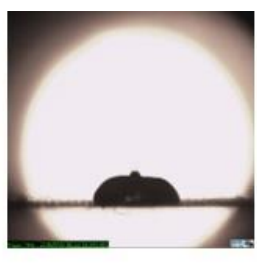

$5.33 \mathrm{~ms}$

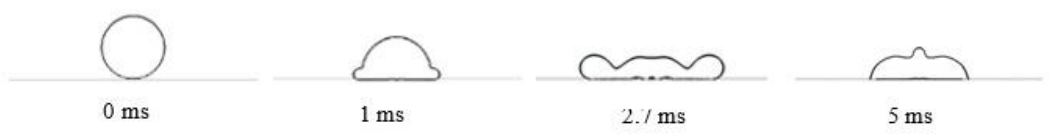

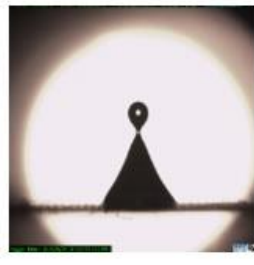

$10 \mathrm{~ms}$

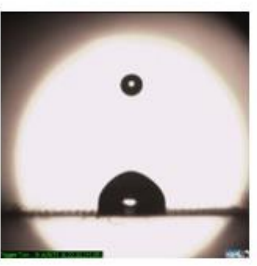

$27 \mathrm{~ms}$

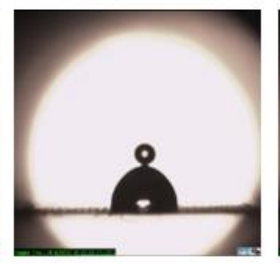

$50.67 \mathrm{~ms}$

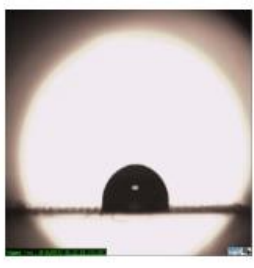

$57 \mathrm{~ms}$
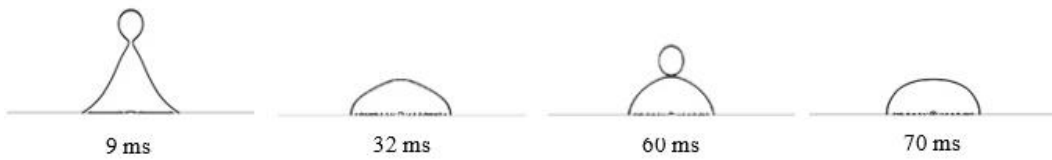

Fig. 2 Deformation processes of the benchmark model

In order to investigate the mechanism of the droplet morphological evolution, pressure field and velocity vector of the benchmark model in Fig. 3 are obtained.

In spreading stage $(0-2.7 \mathrm{~ms})$, the pressure at the center is higher than the edge, forcing the droplet to flow from high pressure to low pressure. The upper velocity of the droplet is substantially downward, and the lower velocity is radial outside, so that the droplet spreads toward the edge $(1 \mathrm{~ms})$. Then the high pressure region gradually shifts to the edge and the velocity at the edge becomes smaller and smaller due to surface tension. At this time, although the three-phase contact line begins to draw back, the droplet at the center still flows to the periphery under the action of inertia, further forming a liquid ring at the edge and reaching the maximum spreading state $(2.7 \mathrm{~ms})$.

In recoiling stage $(2.7-9 \mathrm{~ms})$, affected by the pressure difference and surface tension, the velocity direction at the trailing edge begins to convert toward the center and the pressure rises again, forcing the liquid droplet to retract. At $5 \mathrm{~ms}$, Velocity vectors totally turn around and a strong upward trend forms at the droplet center. The surface energy is converted into kinetic energy, driving the droplet to bounce up quickly.

In oscillating stage $(9-70 \mathrm{~ms})$, the high pressure is gradually formed at the neck of the liquid droplet, leading to the top portion of the droplet overcomes gravity and surface tension ( $9 \mathrm{~ms}$ ). It is the reason why a little bead can be observed in experimenal and numerical results in Fig. 2. Then a little bead separates from the bulk of the droplet and keeps bouncing upward (32 ms). Meanwhile, the bottom velocity points downward under the action of gravity. The bottom of liquid droplet remains oscillating until the little bead falling down to reach final equilibrium state $(70 \mathrm{~ms})$.

Besides, we also found that the pressure is always 
symmetrically distributed during the impacting process, and the high pressure distribution is generally located at the center in porous medium. In pressure field and velocity vector, the obvious bubble entrapment is captured, which cannot be observed in experimenal results [11].

$$
1 \mathrm{~ms}
$$
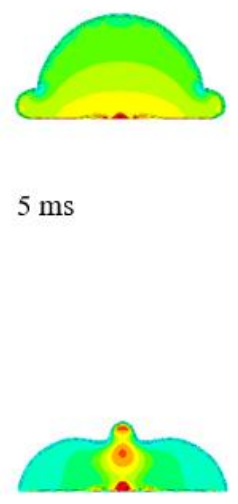

$32 \mathrm{~ms}$

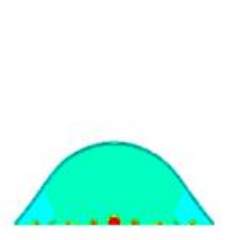

$\bigcirc$
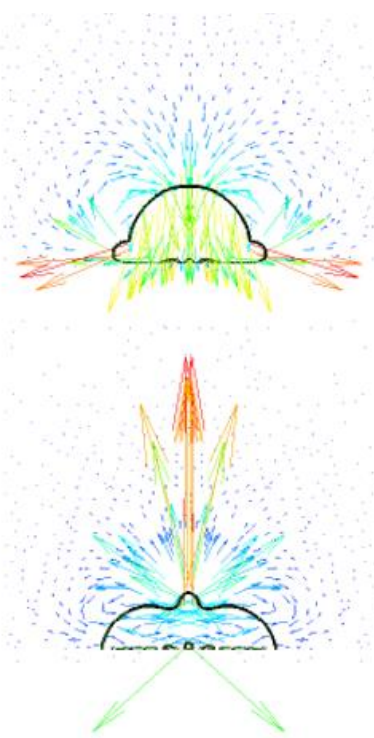

$2.7 \mathrm{~ms}$

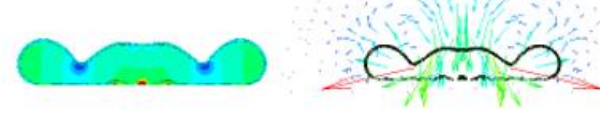

$9 \mathrm{~ms}$
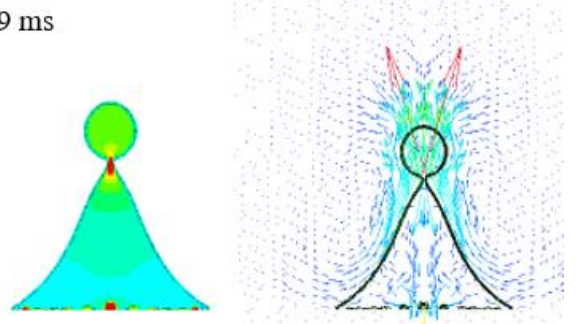

$60 \mathrm{~ms}$
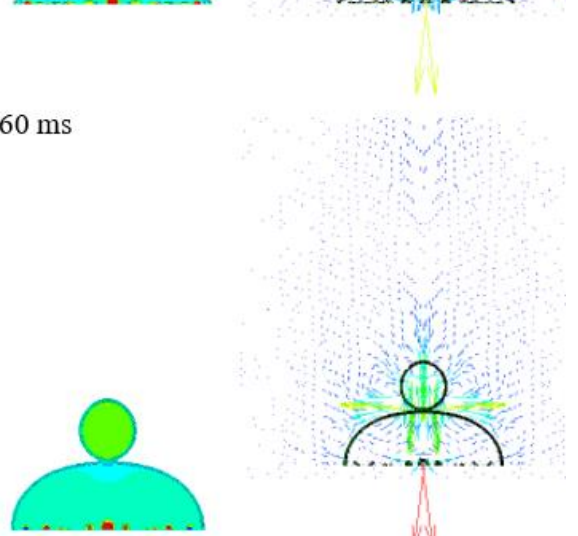

Fig. 3 Pressure field and velocity vector of the benchmark model

\subsection{Spreading factor and contact angle}

Fig. 4 demonstrates the comparison of the numerical and experimental spreading factors of the benchmark model [15]. Two curves are in well agreement and both of them have the similar three stages (spreading, recoiling and oscillating stage). In spreading stage, the spreading factor rises quickly from 0 to peak value, taking almost $2.7 \mathrm{~ms}$ and the maximum spreading factor, which is defined as $\beta_{\max }=D_{\max } / D_{0}\left(D_{\max }\right.$ is the maximum wetting diameter and $D_{0}$ is the initial droplet diameter), is about 1.62 , similar to the experimental peak value $\left(\beta_{\max }=1.61\right)$. Recoiling stage responds to the decrease period from peak value to stabilization stage. In oscillating stage, the spreading factor maintains in a constant.

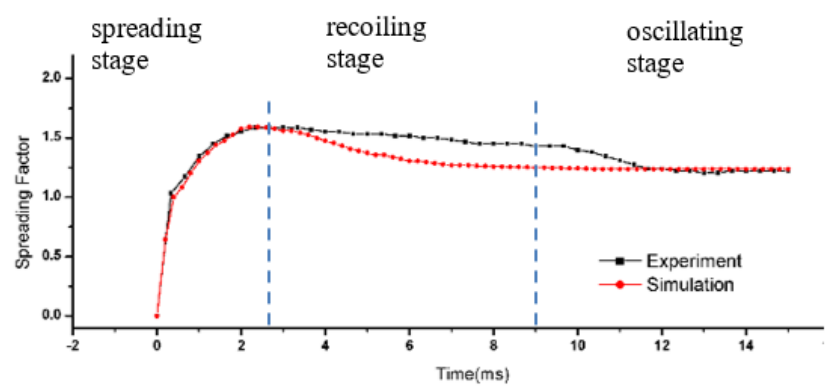

Fig. 4 Spreading factor of numerical and experimental results
In Fig. 5, an entertaining phenomenon is revealed that the contact angle is dynamic while the advancing contact angle $\theta_{A}=144^{\circ}$ in spreading stage, and respectively the receding contact angle $\theta_{R}=55^{\circ}$ in recoiling stage.

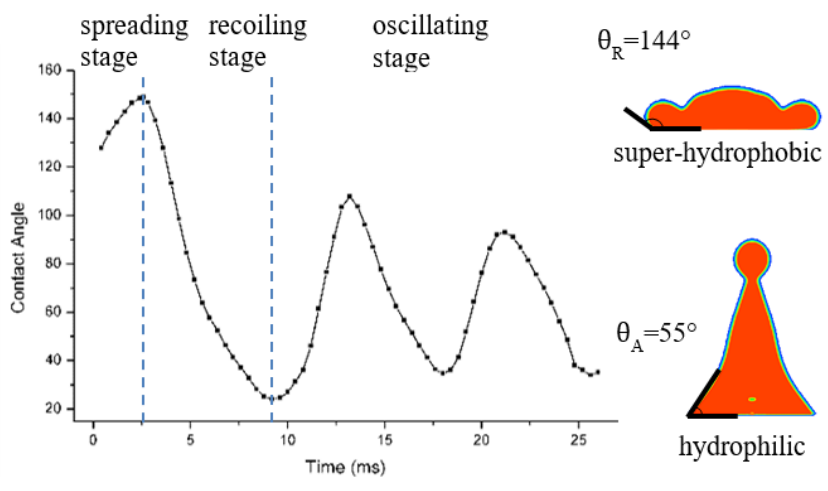

Fig. 5 Contact angle curve of the benchmark model

In order to investigate this phenomenon, we obtain the contact angle curve of the benchmark model at the liquid-porous interface in Fig. 5, response to the morphological evolution of the impacting droplet. In spreading stage, the contact angle increases rapidly to peak value $\left(\theta=149^{\circ}\right)$, while the liquid-porous interface presents super-hydrophobic. As above mentioned, it is the result of the competition between a spreading process of the droplet on the surface 
and an infiltration process inside the porous medium. In this stage, the spreading is stronger than the infiltration due to large kinetic energy and inertia. In recoiling stage, the contact angle decreases to valley value $\left(\theta=25^{\circ}\right)$, and the wettability of the liquid-porous interface shifts from hydrophobic to hydrophilic, because the infiltration gradually surpasses the spreading under the action of surface tension. In oscillating stage, spreading and infiltration are well-matched, and the contact angle keeps oscillating until reaching final equilibrium state.

\subsection{Effect of droplet diameter and impact velocity}

Figs. 6 and 7 shows the effect of different velocities on spreading factors. The droplet diameter is set as 2.12 $\mathrm{mm}$ to investigate the effect of impact velocity, which are $0.75,1.39,1.97$ and $2.42 \mathrm{~m} / \mathrm{s}$, respectively. In Fig. 6 , it is obvious that a higher velocity leads to a higher spreading factor. And all of four lines have a rapid raise stage and slow decline stage, and finally tend to stabilize. Besides, the peak spreading factors of four curves almost occur at the moments of 2.2 2.6 ms, and accordingly the time reaching the stable spreading factors are almost $3.4 \mathrm{~ms}$ for these curves. It is notable that the characteristics on the curve $(v=2.42$ $\mathrm{m} / \mathrm{s}$ ) is not obvious. The reason is the overlarge collision kinetic energy of the droplet, which compels the droplet to splash in spreading process, resulting in the measurement error of the spreading factor.

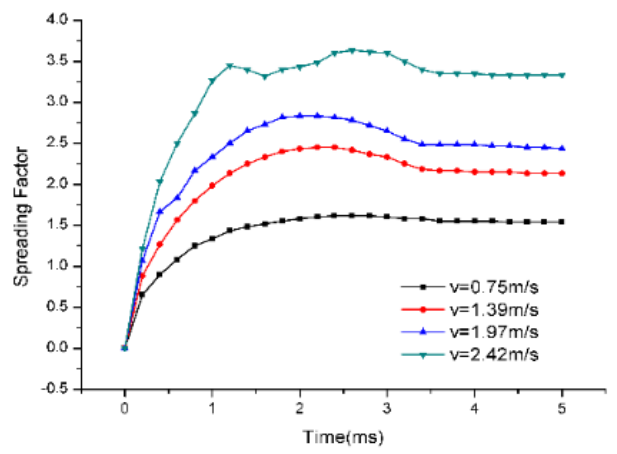

Fig. 6 Maximum spreading factor with different velocities

In Fig. 7, the droplet shapes with different velocities transform dramatically in maximum spreading state. As velocities increasing, the spreading factor rises correspondingly and the droplet shape becomes fractured and thinner. In addition, it can be seen that little liquid drops separate from the liquid body and the size of drops increase as impact velocities rising.

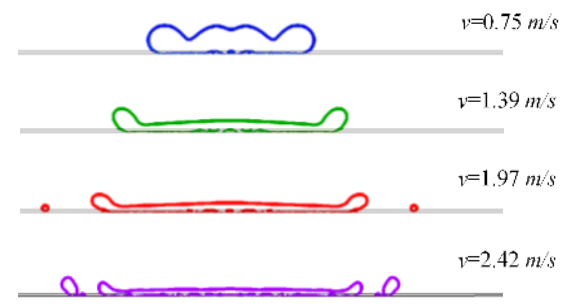

Fig. 7 Droplet shapes with different velocities

Fig. 8 shows that the spreading factor curves of four different diameters, which was set as 2.12, 2.98, 3.54 and $4.58 \mathrm{~mm}$, respectively, with the same droplet velocity of $0.75 \mathrm{~m} / \mathrm{s}$. Apparently, a higher diameter droplet can lead to a higher spreading factor and take more time to reach the stabilization stage. Besides, the time reaching maximum spreading state rises with droplet diameters increasing. It can be seen that the curve $(d=2.12 \mathrm{~mm})$ spends only $3.6 \mathrm{~ms}$ to reach maximum spreading stage, meanwhile it takes 9.6 $\mathrm{ms}$ for the curve $(d=4.58 \mathrm{~mm})$ to stabilize. In Fig. 9, with droplet diameters increasing, the shape of liquid droplets looks similar and maximum spreading factors are correspondingly rising.

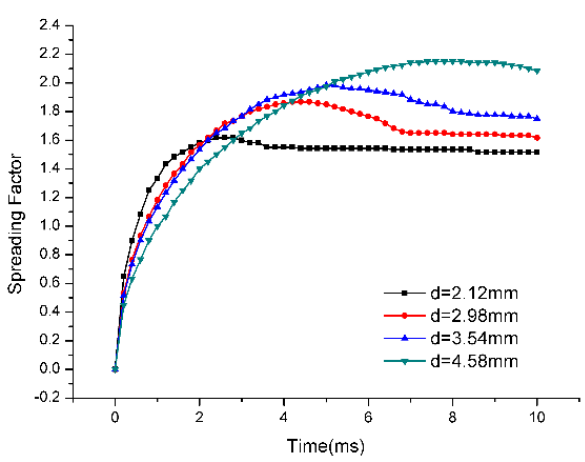

Fig. 8 Maximum spreading factor with different diameters

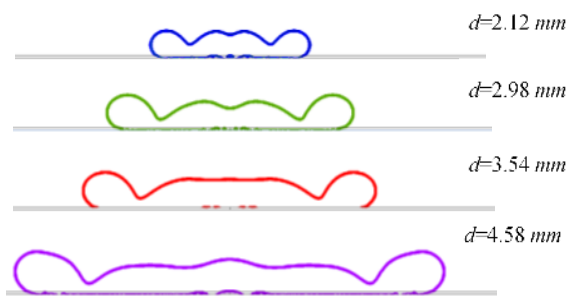

Fig. 9 Droplet shapes with different diameters

\section{Conclusions}

In this approach, the numerical model of the fluid dynamics of liquid droplets impacting on the fabric surface with VOF method is proposed. The following conclusions can be obtained.

1. The results obtained by the proposed model are well in agreement with the experimental results. And pressure distribution and velocity vector are acquired.

2. The obvious bubble entrapment, which cannot be observed in experimenal results, is simulated by the proposed model.

3. The evolution laws of the spreading factor and the contact angle at the liquid-porous interface are acquired. The dynamic of the contact angle is the results of the competition between the droplet spreading on the surface and an infiltration inside the porous medium

4. Droplet diameter increasing leads more dramatic shape distortion of the liquid droplet, and impact velocity increasing leads shorter time to reach the maximum spreading stage.

\section{Acknowledgements}

This work was supported by The Natural Science Foundation of China (No. 11602293).

\section{References}

1. Jugifa S.; Wongpanit N.; Laoketkan T. 2002. The 
combustion of liquid fuels using a porous medium, Experimental Thermal and Fluid Science 26(1): 15-23. https://doi.org/10.1016/S0894-1777(02)00106-1.

2. Zhu C. L.; Li Y. X.; Zhang, Q. 2007. Analysis method of droplet parameters based on rotating multi-cylinder in an ice wind tunnel, Journal of Aerospace Power 22(2): 180-186.

3. Anderson, T. L. 1991. Fracture Mechanics. Fundamentals and Applications, Boca Raton, Ana Arbor: CRC Press. 793 p.

4. Dickey, H.; Watson, V.; Zangelidis, A. 2009. Job satisfaction and quit intentions of offshore workers in the UK North Sea oil and gas industry, Scottish Journal of Political Economy 5(58): 607-633. https://doi.org/10.1111/j.1467-9485.2011.00561.x.

5. Sheng, S. Q.; Bi, F. F.; Guo, Y. L. 2012. Simulation of droplets impact on curved surfaces with lattice Boltzmann method, International Journal of Heat and Mass Transfer 55: 6938-6943.

https://doi.org/10.1016/j.ijheatmasstransfer.2012.07.007.

6. Li, J. Y.; Zhang, B.; Guo, P. H.; Lv, Q. 2014. Impact force of a low speed water droplet colliding on a solid surface, Journal of Applied Physics 116: 214903. https://doi.org/10.1063/1.4903316.

7. Li, J. Y.; Zhang, B.; Guo, P. H.; Lv, Q. 2017. Experimental studies on the effect of Reynolds and Weber numbers on the impact forces of low-speed droplets colliding with a solid surface, Experiments in Fluids 58: 125.

https://doi.org/10.1007/s00348-017-2413-z.

8. Blake, J.; Thompson, D.; Raps, D.; Strobl, T. 2015. Simulating the Freezing of Supercooled Water Droplets Impacting a Cooled Substrate, AIAA Journal 5(7):17251739.

https://doi.org/10.2514/1.J053391.

9. Neyval, C.; Reis, J. R.; Richard, F. G.; Jane, M. 2008. Parametric study of liquid droplets impinging on porous surfaces, Applied Mathematical Modelling 32: 241-261. https://doi.org/10.1016/j.apm.2006.12.006.

10. Neyval, C.; Reis, J. R.; Richard, F. G.; Jane, M. 2004. Numerical simulation of the impact of liquid droplets on porous surfaces, Journal of Computational Physics 197: 747-770. https://doi.org/10.1016/j.jcp.2004.01.024.

11. Golpaygan, A.; Hsu, N.; Ashgriz, N. 2008. Numerical Investigation of Impact and Penetration of a Droplet onto a Porous Substrate, Journal of Porous Media 11(4): 323-341. https://doi.org/10.1615/JPorMedia.v11.i4.10.

12. Kim, W.S.; Lee, S.Y. 2016. Behavior of a water drop impinging on porous substrates examination of contactline drag effect, Atom Sprays 26: 257-74. https://doi.org/10.1615/AtomizSpr.2015012276.

13. Moonhyeok, C.; Gihun, S.; Woosup, S. 2017. A levelset method for droplet impact and penetration into a porous medium, Computers and Fluids 145: 153-166. https://doi.org/10.1016/j.compfluid.2016.12.014.

14. Moonhyeok, C.; Gihun, S.; Woosup, S. 2017. Numerical simulation of droplet impact and evaporation on a porous surface, International Communications in Heat and Mass Transfer 80: 18-27.

https://doi.org/10.1016/j.icheatmastranfer.2016.11.002.

15. Cheng, H.; Qiu, C.; Zhou, C. C.; Sun, X. B.; Yang, R. 2017. Experimental study of spreading characteristics of droplet impacting on canopy fabric surface, Modern Physics Letters B 31: 1750325.

https://doi.org/10.1142/S0217984917503250.

16. Denesuk, M.; Zelinski, B.; Kreidl, N.; Uhlmann, D. 1994. Dynamics of incomplete wetting on porous materials, Colloid Interface Sci 168:142-151.

https://doi.org/10.1006/jcis.1994.1403.

17. Betchen, L.; Straatman, A. G.; Thompson, B. E. 2006. A nonequilibrium finite-volume model for conjugate fluid/porous/solid domains, Numer Heat Transf AAppl 49: 543-65. http://dx.doi.org/10.1080/10407780500430967.

18. Hsu, C. T.; Cheng, P. 1990. Thermal dispersion in a porous medium. Int. J. Heat Mass Transfer 33:15871597.

https://doi.org/10.1016/0017-9310(90)90015-M.

19. Ergun, S. 1952. Fluid flow through packed columns, Chem. Eng. Prog. 48: 89-94.

20. Hadin, A. 1994. Forced convection in a porous channel with localised heat sources, Heat Transfer 116: 465-472. https://doi.org/10.1115/1.2911419.

Qilei Guo, Peng Sun, Chen Chen, Yao Ma

NUMERICAL STUDY OF LOW-SPEED DROPLETS IMPACTING ON THE FABRIC SURFACE

S u m m a r y

In this approach, the numerical model of the fluid dynamics of liquid droplets impacting on the fabric surface with VOF method is proposed. The results obtained by the proposed model are well in agreement with the experimental results. The mechanism of the liquid droplet morphological evolution is investigated by pressure distribution and velocity vector, and the obvious bubble entrapment, which cannot be observed in experimenal results, is captured by the proposed model. The evolution laws of the spreading factor and the contact angle at the liquid-porous interface are obtained and the reason why the contact angle is dynamic is analyzed. The effects of droplet diameter and impact velocity on the fluid flow characteristics are also discussed. Usually, droplet diameter increasing leads more dramatic shape distortion of the liquid droplet, and impact velocity increasing leads shorter time to reach the maximum spreading stage. Finally, based on the properties of the impacting droplet by the proposed model, the important references of optimizing the parachute design for severe weather are built.

Keywords: liquid droplets; fabric surface; impacting; volume of fraction; multiphase flow.

Received March 14, 2019 Accepted November 21, 2019 